\title{
Laser-driven micro-explosive bonding of aluminium films to copper and silicon
}

\author{
DALE E. ALEXANDER, GARY S. WAS \\ Department of Nuclear Engineering, University of Michigan, Ann Arbor, Michigan 48109, \\ USA
}

FRED J. MAYER

KMS Fusion Inc., 3621 S. State, Ann Arbor, Michigan 48104, USA

Laser explosive microfabrication was used to bond micrometre-thick aluminium films to both copper and silicon substrates. Bonding was observed in both systems for laser intensities greater than about $1 \times 10^{9} \mathrm{~W} \mathrm{~cm}^{-2}$ and resulted in contiguous, uniform films when performed in a rough vacuum of 25 to 70 millitorr. At intensities greater than $9 \times 10^{9} \mathrm{~W} \mathrm{~cm}^{-2}$ the transferred films were generally black in colour due to oxidation in the aluminium-on-copper shots and due to substrate vaporization in the aluminium-on-silicon shots. SEM examination of the bond interfaces indicated mixing of film and substrate through wave formation and possibly through liquid-phase mixing due to thermal conduction of the laser pulse. Tape adhesion testing of the laser-bonded films showed a significant increase in adhesion over vapour-deposited films in both $\mathrm{Al}-\mathrm{Cu}$ and $\mathrm{Al}-\mathrm{Si}$. Vapour-deposited films were easily removed in their entirety by a single tape pull while metallic portions of the laser-bonded films resisted removal by the tape in all but two tests. The enhanced adhesion evident in the laser-bonded films was a result of intermixing observed at the film-substrate interface.

\section{Introduction}

Explosive bonding is a well-established large-scale technique [1] used extensively in industry to bond a thin layer of a more noble and costly metal to a heavier and less costly load-bearing substrate. The process permits the bonding of metals that would normally be incompatible using conventional welding techniques. In conventional explosive bonding, the thin layer of metal flyer plate to be bonded to the substrate is impacted on to the substrate at high velocities via an explosive sheet charge. Successful bonding is critically dependent on parameters such as the stand-off distance, flyer plate velocity, and angle of inclination between the flyer plate and substrate, all of which are material-dependent.

Explosive bonding has also been accomplished using lasers. The work of Frish and Nebolsine [2] demonstrated the ability of pulsed laser beams to bond metal foils to substrates. The foils were generally $25 \mu \mathrm{m}$ thick and were placed in direct contact with the substrate prior to irradiation. The laser pulse incident on the foil explosively vaporized a surface layer, resulting in pressure-assisted bonding of the foil to the substrate.

On a smaller scale explosions can be initiated via laser explosive microfabrication (LEM). The LEM technique, as defined here, utilizes a focused, pulsed laser beam incident on the back side of a transparent substrate containing an opaque metal film. The laser pulse rapidly heats and "explodes" the film from the glass substrate preferentially along the axis perpendicular to the substrate. This technique, in varying forms, has been used for a number of applications including plasma generation [3], photomask repair $[4,5]$, flyer film propulsion studies [6], and $p-n$ junction formation [7].

Using the LEM technique it should be possible to scale down the explosive bonding process (Fig. 1). The concept is similar to laser-driven flyer film experiments [6], except that here the interest is in the material bonding produced as a result of a driven film impacting on a substrate. In Fig. 1a the target arrangement is illustrated; one of the two metal films vapourdeposited on a thick glass slide is the same as a flyer plate (or flyer film in this case) and the other film serves as the exploding film. The latter is vaporized by the incident laser pulse and provides the pressure to propel the flyer film away from the glass and impact it on the substrate (Fig. $1 \mathrm{~b}$ to $\mathrm{d}$ ).

In this work the LEM process was used to bond thin ( $1.0 \mu \mathrm{m}$ thick) metal films to both metal and semiconductor substrates. The films were placed in direct contact with the substrate prior to irradiation and thus the approach was similar to that of Frish and Nebolsine mentioned above. However, this experiment differed in that (i) the films bonded were much thinner, and (ii) the tamping effect of the glass slide was expected to significantly increase the pressure on the film. Aluminium film bonding to copper was studied since previous work $[2,8,9]$ had demonstrated 


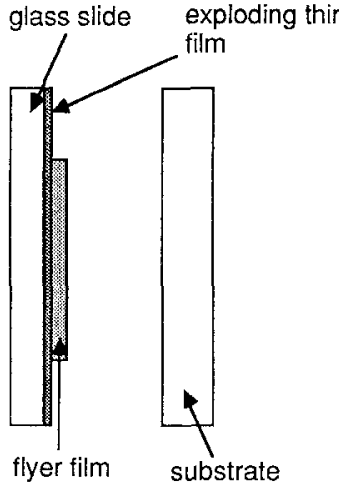

(a)
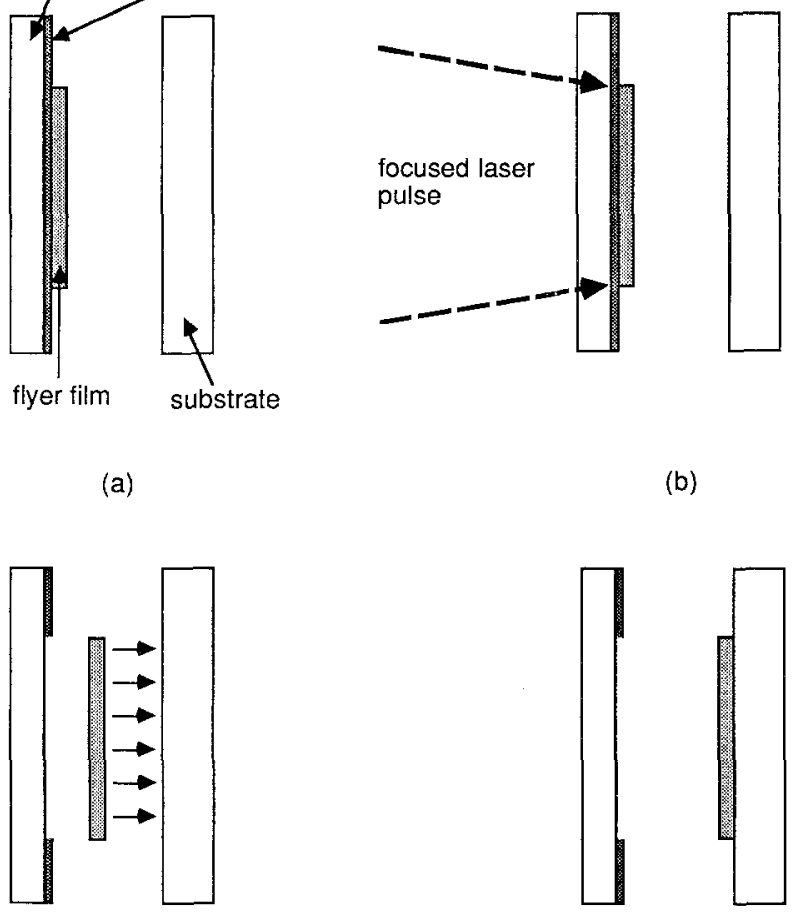

(c)

(d)

Figure 1 Small-scale LEM explosive bonding process: (a) side view of target prior to laser irradiation; (b) laser pulse incident on target; (c) flyer film propelled toward substrate; (d) flyer film impacts on substrate thus bonding it to substrate.

the relative ease of explosive bonding of these metals. Aluminium film bonding to silicon was also examined.

\section{Laser explosive microfabrication process}

In conventional explosive bonding, two parameters in particular are critical for good bonding: (i) the flyer plate velocity, and (ii) the angle of inclination between the flyer plate and the substrate. The second of these parameters is controlled by the orientation of the flyer plate with respect to the substrate. The first parameter can be related to the incident laser intensity by a simple conservation-of-energy argument. Such an analysis has been experimentally supported by velocity measurements of laser-driven flyers [6]. The LEM process can then be controlled to yield velocities in the required range for bonding.

Consider an incident laser pulse of energy $E_{\mathrm{L}}$, pulse length $\tau$, and focused diameter $D$. The pulse is incident on an LEM target as illustrated in Fig. 1b. The target consists of the exploding film and the flyer film, the latter having density $\varrho$. The incident pulse vaporizes the exploding film and propels the flyer film with a velocity $v$. Of the incident laser energy, only a fraction, $\varepsilon$, is converted into kinetic energy of the flyer film. For a given material only a certain range of velocities permits good bonding to occur. Such a criterion is expressed as $v_{\min } \leqslant v \leqslant v_{\max }$, where $v_{\min }$ and $v_{\max }$ are respectively the minimum and maximum flyer film velocities permitting bonding [8].
Using conservation of energy, the LEM process is described as

$$
\varepsilon E_{\mathrm{L}}=\frac{1}{2} m v^{2}
$$

where $m$ is the mass of the flyer film. Since the flyer film is of the same diameter as the incident laser pulse, Equation 1 can be rewritten eliminating $m$ as

$$
\varepsilon E_{\mathrm{L}}=\frac{1}{2} \frac{\varrho t \pi D^{2}}{4} v^{2}
$$

where $t$ is the thickness of the flyer film.

Laser intensity, $I$, is expressed as

$$
I=\frac{4 E_{\mathrm{L}}}{\pi D^{2} \tau} .
$$

Substituting the value for $E_{\mathrm{L}}$ from Equation 2 into Equation 3 yields

$$
I=\frac{\varrho v^{2}}{2 \tau \varepsilon} t
$$

For a given choice of laser and material, $\varrho, \tau$, and $\varepsilon$ are fixed. Further, if $v$ is restricted to the range of velocities permitting explosive bonding, Equation 4 gives the incident laser intensity, $I$, required to bond a film of thickness $t$.

Unknown in this derivation is the value of $\varepsilon$, the efficiency of laser energy conversion to kinetic energy. An upper bound on $\varepsilon$ is the absorption coefficient of the exploding metal film for the laser light wavelength of interest $(1.06 \mu \mathrm{m})$ yielding $\varepsilon \approx 0.1$. However, less ideal circumstances dictate a smaller value for $\varepsilon$. The following analysis considered two values: $\varepsilon=0.1$ and $\varepsilon=0.01$.

In addition to the velocity criterion, the angle of inclination of the flyer plate with respect to the substrate is of importance. This criterion arises out of the need for the "jetting phenomenon" to clean metal surfaces prior to contact. As the plate and the substrate impact at high velocities, a jet consisting of surface metal from both pieces escapes from the point of contact, due to the large forces and high temperatures experienced in the immediate area of impact [1]. It is likely that the jetting phenomenon is of little significance in the scaled-down version of explosive bonding because the materials to be bonded already have clean, highly polished surfaces and the experiments are typically performed in a vacuum. Therefore the angle between the flyer film and the substrate is set to zero.

Two materials of interest in the proposed bonding experiments are aluminium and copper. Equation 4 is applied to these materials to determine the range of laser intensity and thickness over which bonding is expected. The values for $v_{\min }$ and $v_{\max }$ for copper are 70 and $187 \mathrm{~m} \mathrm{sec}^{-1}$, respectively, and for aluminium are 182 and $541 \mathrm{~m} \mathrm{sec}^{-1}[8]$. The laser pulse length, $\tau$, was set to $1.0 \mathrm{nsec}$. Calculations for aluminium using Equation 4 are graphically illustrated in Fig. 2, which shows the laser intensity range over which bonding is expected. For a film thickness of $1 \mu \mathrm{m}$, the lower limit of this intensity range is $4.5 \times 10^{7} \mathrm{~W} \mathrm{~cm}^{-2}$, for $v=v_{\min }$ and $\varepsilon=0.1$, and the upper limit is $4.0 \times$ $10^{9} \mathrm{~W} \mathrm{~cm}^{-2}$, for $v=v_{\max }$ and $\varepsilon=0.01$. 


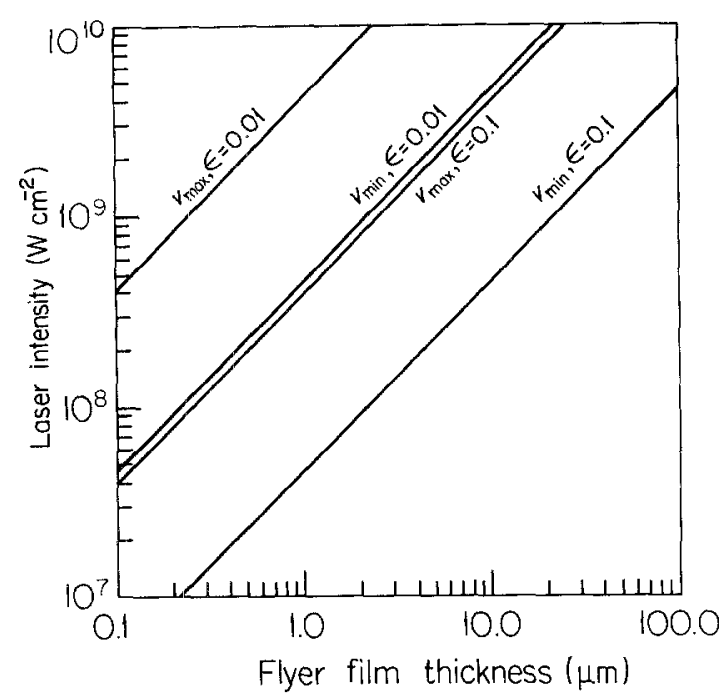

Figure 2 Relationship between film thickness and laser intensity required for bonding aluminium films as predicted for a scaleddown explosive bonding experiment. $v_{\min }$ and $v_{\max }$ are respectively the minimum and maximum flyer plate velocities required for bonding (for aluminium, $v_{\min }=182 \mathrm{~m} \mathrm{sec}^{-1}$ and $v_{\max }=541 \mathrm{~m} \mathrm{sec}^{-1}$ ). $\varepsilon$ is the efficiency of laser energy transfer.

\section{Experimental procedure}

Copper substrates consisted of $1 \mathrm{~cm}$ square pieces cut from sheet stock and mounted in Bakelite ${ }^{\circledR}$. To ensure a smooth interface for bonding, these samples were ground with 180 to 600 grit $\mathrm{SiC}$ paper. Polishing was done with an alumina slurry followed by Synton ${ }^{\circledR}$ as a final polish. Polished silicon substrates were $0.254 \mathrm{~mm}$ thick, boron-doped and oriented in the $\left[\begin{array}{ll}10 & 0\end{array}\right]$ direction.

Thin films of aluminium were prepared in vacuum by vapour deposition on square pieces of glass slides of approximately $1 \mathrm{~mm}$ thickness in a vacuum of about $4 \times 10^{-6}$ torr. A $0.5 \mu \mathrm{m}$ layer was first deposited on the slide. This aluminium layer served as the "exploding film". On top of this film the "bonding film" was deposited, consisting of $3 \mathrm{~mm}$ diameter, $1.0 \mu \mathrm{m}$ thick spots of aluminium. The deposited glass slides were placed on to the substrates with the $3 \mathrm{~mm}$ spots in direct contact with the substrate. The constructed target was then placed on an $X-Y-Z$ manipulator and into a target chamber.

An Nd-YAG laser with a wavelength of $1.06 \mu \mathrm{m}$ and pulse length of $1 \mathrm{nsec}$ was used to perform target shots. Because the required laser intensity was well below that nominally delivered by the laser, the experiment was "piggy-backed" in such a way as to use the laser light reflected from a polarizer plate during passage of the main pulse (Fig. 3). The arrangement provided up to $20 \mathrm{~J}$ of energy incident on the first surface of the glass substrate. The reflected laser pulse was focused on to the target located in the target chamber.

Shots were made on $31 \mathrm{Au}-\mathrm{Cu}$ targets in air. The incident laser intensities for these shots were varied from $8.8 \times 10^{8}$ to $1.1 \times 10^{10} \mathrm{~W} \mathrm{~cm}^{-2}$. For Al-Si, 12 shots were performed in a rough vacuum ranging from 25 to 70 millitorr. Incident laser intensities were varied from $1.6 \times 10^{9}$ to $1.3 \times 10^{10} \mathrm{~W} \mathrm{~cm}^{-2}$. Temperature and humidity affected the energy output of the laser and thus reduced the ability to predict the incident laser intensity in advance.

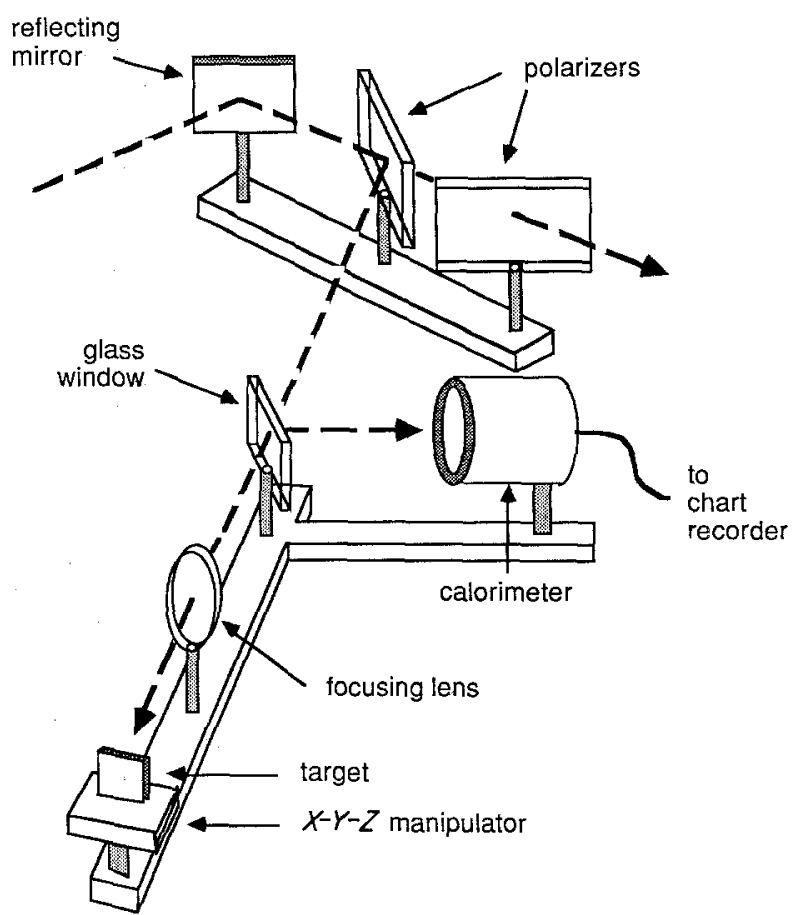

Figure 3 Schematic diagram of experimental arrangement used in LEM bonding experiments. The dashed line indicates the laser pulse path. The target chamber is not pictured.

The adhesive strength of the bonded films was tested via the "Scotch Tape" test [10]. 3M Scotch Brand Magic Transparent Tape ${ }^{\circledR}$ was placed firmly down on top of the bonded spots. The tape was then pulled back slowly by hand, taking care to keep the tape perpendicular to the substrate. Upon removal, less adherent material became detached from the substrate. For comparison, the test was performed on a number of spots of the same thickness which had been directly vapour-deposited on to the substrates.

After adhesion testing, the structure of the laserbonded films was examined. Specimens were sectioned transverse to the interface and examined using optical and scanning electron microscopy (SEM). Once sectioned, the samples were remounted to permit grinding and polishing of the interface region. Imaging in the SEM was conducted using both secondary electrons and back-scattered electrons. SEM X-ray mapping was also employed.

The Scotch Tape test was selected since it provided a simple, standardized test for comparing adhesion observed in vapour deposition with adhesion observed in the laser-irradiated films. More quantitative adhesion tests considered for use included the scratch test [11] and the pull test [12]. However, both suffer from shortcomings associated with test interpretation or reproducibility. The scratch test uses a metal point drawn across a film under a load applied normal to the surface. Adhesion is measured by the critical load required to detach the film from the surface. Disagreement exists, however, concerning how the onset of film detachment is measured [13]. The pull test measures the tensile stress required to detach a film which is pulled via a rod attached to the film. However, a practical problem exists in attaching pull rods to the bonded films and therefore in the reproducibility of 

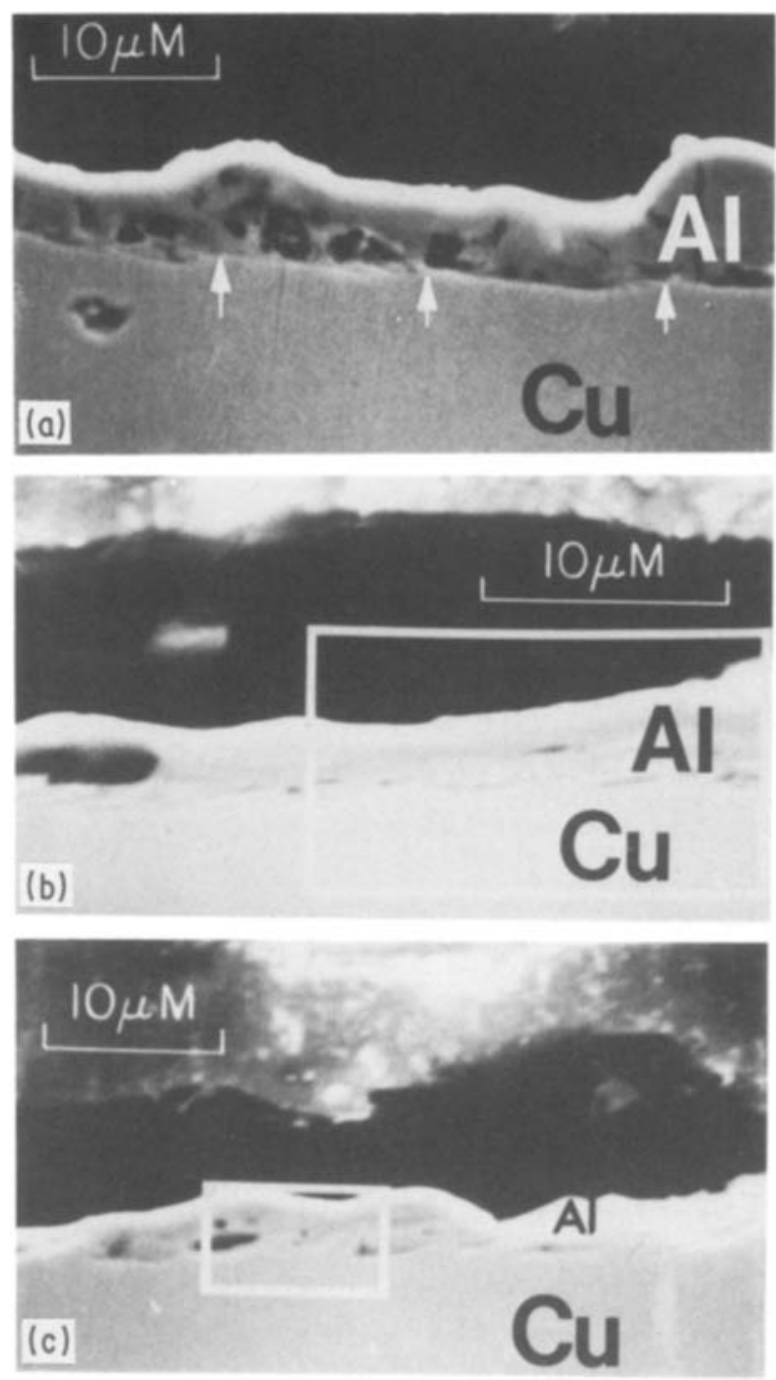

Figure 4 Scanning electron micrographs showing the interfaces of aluminium films bonded to copper substrates using the LEM technique. (a) Arrows indicate crests of waves at film-substrate interface. (b) A flatter wave structure observed in boxed region. (c) Sharp pronounced waves observed in boxed region.

results [14]. For these reasons both of these tests were eliminated from consideration.

\section{Results and discussion}

\subsection{Al-Cu targets}

Transfer of aluminium film to the copper substrate occurred only for intensities greater than $1.0 \times 10^{9} \mathrm{~W}$ $\mathrm{cm}^{-2}$. Below this intensity little, if any, aluminium was transferred from the glass slide to the copper. In these low-intensity cases, however, some disturbance of aluminium on the glass slide was evident after the slide was removed from the copper substrate. In the intensity range of 1 to $9 \times 10^{9} \mathrm{~W} \mathrm{~cm}^{-2}$, the transferred films were metallic in appearance. At intensities greater than $9.0 \times 10^{9} \mathrm{~W} \mathrm{~cm}^{-2}$ the transferred films were generally black in appearance due to oxidation by the high laser intensities. In some cases, bubbling was also observed.

The structure of the aluminium films laser-bonded to copper are shown in the SEM photographs in Fig. 4. In general, the films viewed in this cross-sectional manner show thick (on the order of micrometres), isolated segments of aluminium on the copper substrate. These segments are no greater than 30 to $80 \mu \mathrm{m}$ in length. Holes are observed within the aluminium film which resulted from air entrapment between the flyer film and substrate.

Observation of the interface between the aluminium and the copper shows that intermixing of the two metals has occurred. The photographs in Fig. 4 show a somewhat regular wave-like pattern across the interface. Such a characteristic is similar to the bonds formed in explosive welding. Fig. 4a shows a region of aluminium film about $4 \mu \mathrm{m}$ in thickness in which very flat waves are observed at the interface (indicated by arrows), spaced quite regularly apart at about $10 \mu \mathrm{m}$. In Fig. $4 \mathrm{~b}$ an aluminium film is pictured with similar thickness to that in Fig. 4a but possessing better thickness uniformity. Again the flat waves are observed, particularly in the region enclosed by the box in the photograph. Fig. $4 \mathrm{c}$ shows more pronounced waves at the interface in the region enclosed by the box. These waves are observed with amplitudes on the order of $1.0 \mu \mathrm{m}$.

Adhesion testing of the laser-bonded aluminium films on copper indicated a significant improvement in adhesion compared to vapour-deposited films. Tape pulls performed on the vapour-deposited films readily detached all the films tested in their entirety. For the laser-bonded films only the black areas of the films had material removed by the tape. These areas corresponded to regions where the laser intensity exceeded $9 \times 10^{10} \mathrm{~W} \mathrm{~cm}^{-2}$. The material removed was of a black, fine, powder-like consistency. The tape failed to remove the transferred metallic aluminium films in all cases but one. The one exception occurred for a film bonded at a laser intensity of $8.5 \times 10^{9} \mathrm{~W} \mathrm{~cm}^{-2}$. In this case, approximately $10 \%$ of the area of the metallic film was removed.

These observations (particularly the observation of air entrapment) lend support to the conduction mechanism of heat transfer of the laser pulse, resulting in the melting and mixing of the metals at the bond interface. The fact that the aluminium flyer film was in direct contact with the substrate allows the transfer of heat through the exploding film, flyer film, and substrate. Melting and re-solidification of the aluminium film permits the entrapment of air as observed.

If the assumption is made that the aluminium film remains in the solid state, an expression for the timedependent temperature profile can be given which can be used to estimate the temperature at the interface of the aluminium and copper. Although the film does not remain solid, it is instructive to consider this problem for yielding an upper bound on the temperature at the film-substrate interface. The temperature profile is expressed as [15]

$$
T(z, t)=T_{0}\left(\frac{t_{\mathrm{p}}}{t}\right)^{1 / 2} \exp \left(\frac{-z^{2}}{4 D t}\right)
$$

where $z$ is the distance into the surface and $t$ is the time in seconds after the laser pulse. Taking $z=1.5 \mu \mathrm{m}$, it can be shown that the temperature reaches its maximum of $929^{\circ} \mathrm{C}$ at $15 \mathrm{nsec}$. This temperature is above the melting point of aluminium $\left(660^{\circ} \mathrm{C}\right)$, but is still below the melting point of copper $\left(1084^{\circ} \mathrm{C}\right)$. 


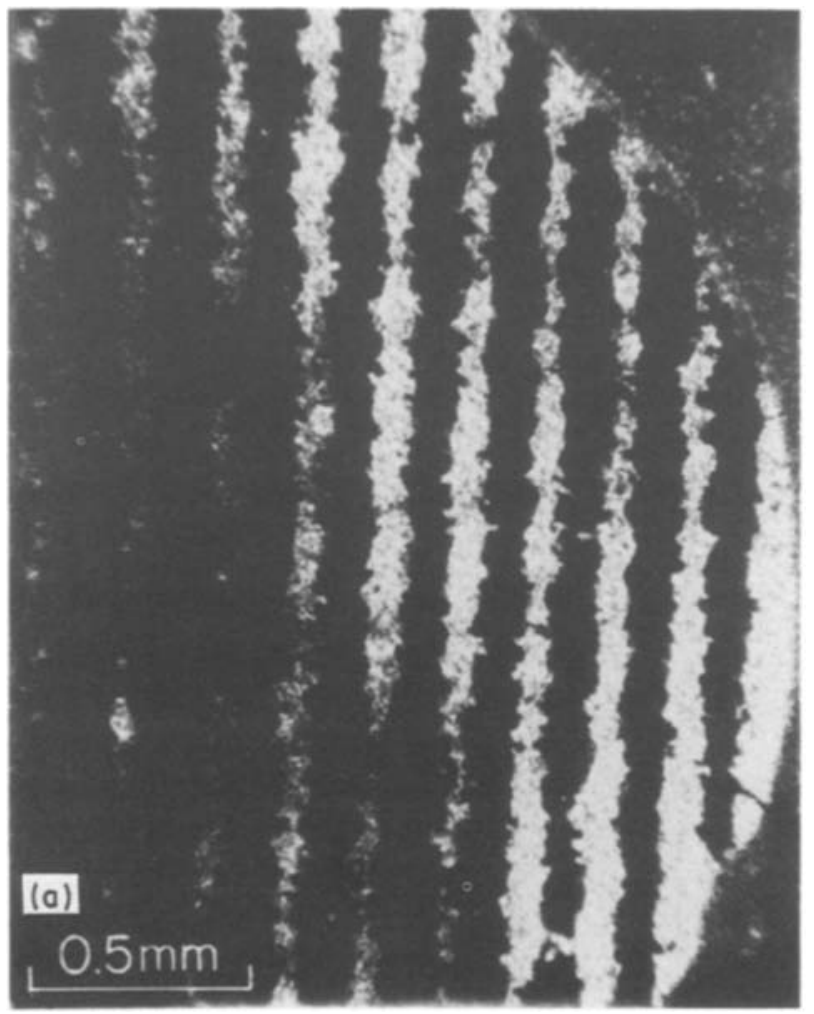

In the experiment, the interface temperature is expected to be lower than the calculated value since the aluminium did not remain in the solid state. The high temperatures result in partial film vaporization and partial melting. Heat is expended in these phase transformations and is not available for transfer to the interface. Also, the effective thermal conductivity will be decreased by the gas and liquid phases which in turn will inhibit heat transfer to the interface. Based on these considerations, it appears that melting of the copper probably did not occur to a significant extent.

Another concern that must be addressed, however, is the effect of alloying on the reduction of the melting temperature required at the interface. If the pressurewave induced by the exploding aluminium film provided slight hydrodynamic mixing of the aluminium and copper at the interface, the alloy would have a reduced melting point. The temperature at the interface would not have to exceed the melting point of copper, but rather melting could occur at some intermediate temperature between the copper and aluminium melting points, dependent on the composition at the interface. In the light of this alloying consideration, it is evident that liquid-state mixing cannot be ignored in this experiment.

\subsection{Al-Si targets}

Transfer of aluminium film to the silicon substrates occurred for intensities greater than $1.6 \times 10^{9} \mathrm{~W}$ $\mathrm{cm}^{-2}$. Fig. 5a shows a micrograph of a portion of a typical bonded aluminium spot taken at low magnification in an optical microscope. The round edges of the spot are very distinct. Also, the areas where the aluminium transferred are bright, smooth and contiguous. These observations are in contrast to the piecewise transfer of aluminium to copper observed for the laser shots performed in air. The use of a rough

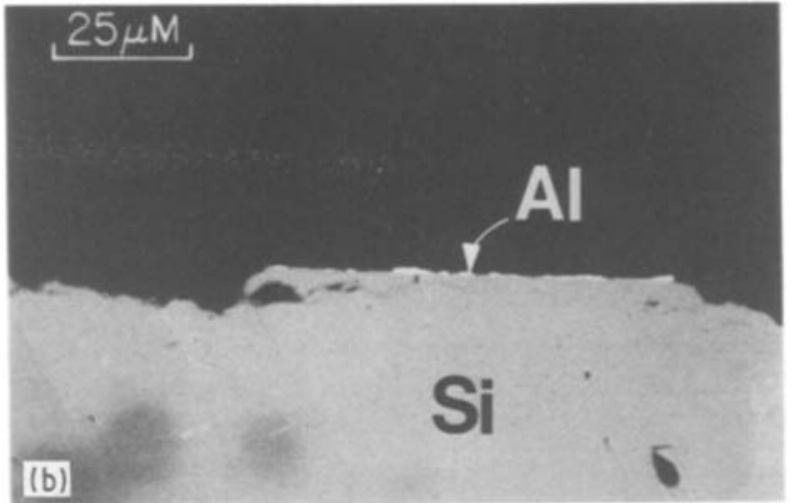

Figure 5 (a) Optical micrograph of part of a $3 \mathrm{~mm}$ diameter spot deposited on silicon using the LEM process. The banded structure is due to the transverse variation in laser intensity. (b) Optical micrograph of $\mathrm{Al}-\mathrm{Si}$ interface at a transverse cut to the bands seen in (a). Irregular but contiguous aluminium film seen on top of the plateau.

vacuum thus improved the structure of the transferred film.

Evident in Fig. 5a is a band structure in which the light metallic bands are approximately $150 \mu \mathrm{m}$ in width. Although they appear straight, the bands are actually arcs of large concentric circles which correspond to transverse intensity variations in the focused laser spot. The light bands correspond to the bonded aluminium film while the dark bands are damaged silicon. In these latter bands the laser intensity was sufficiently high to actually vaporize the silicon to a certain depth, giving it the black colour seen in the photo. As incident intensities were increased to greater than $9.0 \times 10^{9} \mathrm{~W} \mathrm{~cm}^{-2}$, this damaged area encompassed the entire area of the transferred film, giving it a dark grey to black colour.

Interfacial examination of bonds for the $\mathrm{Al}-\mathrm{Si}$ targets was performed using both optical microscopy and the SEM. Fig. 5b is an optical micrograph of the film substrate interface transverse to the band structure observed in Fig. $5 \mathrm{a}$. To the left, the damaged silicon valley is observed which corresponds to the dark bands of Fig. 5a. The laser intensity was sufficiently high to vaporize the surface of the silicon in this region. To the right, the bright aluminium film bonded to the plateau is readily apparent. This region corresponds to the bright bands in Fig. 5a. Although the film is irregular in thickness, it is contiguous across the length of the plateau.

Adhesion testing of the laser-bonded aluminium films on silicon indicated a significant improvement in adhesion compared to vapour-deposited films. Tape pulls performed on the vapour-deposited spots caused the entire film to detach quite easily, identical with results observed for $\mathrm{Al}-\mathrm{Cu}$. As with the $\mathrm{Al}-\mathrm{Cu}$ shots, the only material removed by the tape pulls was from the black areas of the laser-bonded films. Again, this material was in the form of a fine, black powder consisting in part of damaged silicon. Only slight removal of the metallic film was observed in one test. Approximately $5 \%$ of the area was removed from a film bonded at an intensity of $5 \times 10^{9} \mathrm{~W} \mathrm{~cm}^{-2}$. In all 
other cases, no portions of the bonded aluminium films were removed.

Operative also for the Al-Si target shots was the mechanism of heat transfer through to the substrate as described above. As was the case in the $\mathrm{Al}-\mathrm{Cu}$ targets, the calculated temperature at the interface, $929^{\circ} \mathrm{C}$, does not exceed the melting point of silicon $\left(1410^{\circ} \mathrm{C}\right)$; however, the alloying effect mentioned previously could permit interfacial melting and liquid mixing. Thus the possibility exists that under the conditions of Al-Si bonding, quite extensive melting and intermixing of the aluminium and silicon occurred. In such a case, the physically distinct bond interfaces formed in the $\mathrm{Al}-\mathrm{Cu}$ shots would be absent. In their place, a continuous mixture, ranging in composition from pure aluminium to pure silicon, may have constituted the bond. Such a phenomenon has been verified by secondary-ion mass spectrometry measurements performed on low-intensity laser-irradiated thin aluminium films on silicon [7].

\section{Summary}

This experiment demonstrated the use of the LEM process to bond micrometre-thick films of aluminium to both copper and silicon substrates. Bonding was observed in both systems for laser intensities greater than about $1.0 \times 10^{9} \mathrm{~W} \mathrm{~cm}^{-2}$ and resulted in films with distinct spot edges when performed in at least a rough vacuum ( 25 to 70 millitorr). This was particularly evident in the $\mathrm{Al}-\mathrm{Si}$ shots in which large, contiguous films were bonded. At intensities greater than $9.0 \times 10^{9} \mathrm{~W} \mathrm{~cm}^{-2}$ the transferred films were generally black in appearance due to oxidation in the $\mathrm{Al}-\mathrm{Cu}$ shots and due to substrate vaporization in the $\mathrm{Al}-\mathrm{Si}$ shots.

SEM observation of the film-substrate interface for the Al-Cu targets showed significant intermixing of the two metals. In some cases, a wavy-type interface was observed which is typical of large-scale explosive bonding bonds. The intermixing accounts for the observed enhanced adhesion of the laser-bonded films. Optical microscopy of the Al-Si interfaces provided evidence for long, contiguous film bonding.

The laser-bonded films demonstrated a significant increase in adhesion over vapour deposited films when tested using the Scotch Tape adhesion test. Vapourdeposited spots were easily removed with just one pull of the tape, while metallic portions of the laser- bonded films resisted removal by the tape in all but two instances.

Since the bonded film was in direct contact with the substrate, consideration must be given to the role of conduction heat transfer in the bonding process. The incident laser pulse probably resulted in melting at and below the interface of the film and substrate. Thus, in addition to hydrodynamic mixing from the pressure induced on the interface of the metals due to the exploding film, high temperatures may have permitted thermal mixing to occur.

\section{Acknowledgement}

The authors gratefully acknowledge the support for D. Alexander by the Nuclear Engineering, Health Physics, and Radioactive Waste Management Fellowship programme administered by Oak Ridge Associated Universities for the US Department of Energy.

\section{References}

1. "Explosive Welding", Mech. Eng. (May 1978) 28.

2. M. B. FRISH and P. NEBOLSINE, "Surface Coating and Alloying by Laser Induced Heat and Pressure", Final Report, NSF Grant No. DMR-8260087 (1983).

3. F. J. MAYER and G. E. BUSCH, J. Appl. Phys. 57 (1985) 827

4. M. HONGO and J. NAKABAYSHI, US Patent 4190759 (1980).

5. T. J. LANE and J. J. WYNNE, IBM Tech. Disclosure Bull. 27 (1984) 446.

6. S. A. SHEFFIELD, J. W. ROGERS and J. N. CAST. ANEDA, in Proceedings of American Physical Society Fourth Topical Conference on Shock Waves in Condensed Matter (Plenum, New York, 1985) p. 541.

7. M. TOULEMONDE, J. C. MULLER and R. STUCK, J. Solar Energy Eng. 108 (1986) 103.

8. S. H. CARPENTER and R. H. WITTMAN, Ann. Rev. Mater. Soc. 5 (1975) 177.

9. A. OBERG, N. MARTENSSON and J. A. SCHWEITZ, Met. Trans. A 16 (1985) 841.

10. D. S. CAMPBELL, in "Handbook of Thin Film Technology", edited by L. I. Maissel and R. Glang (McGraw-Hill, New York, 1970) Ch. 12.

11. O. S. HEAVENS, J. Phys. Radium 11 (1950) 385.

12. B. N. CHAPMAN, J. Vac. Sci. Tech. 11 (1974) 106.

13. J. E. GREENE, J. WOODHOUSE and M. PESTES, Rev. Sci. Instr. 45 (1974) 747.

14. C. WEAVER, J. Vac. Sci. Tech. 12 (1975) 18.

15. M. BASS, in "Physical Processes in Laser-Materials Interactions", edited by M. Bertolotti (Plenum, New York, 1984).

Received 26 June

and accepted 22 September 1987 